\title{
Analyses des paramètres démographiques et zootechniques du cheptel bovin des élevages péri-urbains laitiers de la ville de Bobo-Dioulasso à l'Ouest du Burkina Faso
}

\author{
Nouhoun ZAMPALIGRE ${ }^{1,2^{*}}$, Inoussa SAVADOGO ${ }^{2,3}$ et Mamadou SANGARE ${ }^{2}$ \\ ${ }^{I}$ Institut de l'Environnement et de Recherches Agricoles, Station de Farako-ba, Bobo Dioulasso, Burkina Faso. \\ ${ }^{2}$ Université Nazi Boni, Bobo Dioulasso, Burkina Faso. \\ ${ }^{3}$ Centre International de Recherche-Développement sur l'Elevage en Zone Subhumide, Bobo Dioulasso, \\ Burkina Faso. \\ *Auteur correspondant ; E-mail : nouhoun@gmail.com
}

\section{RESUME}

Les statistiques sur les paramètres démographiques et zootechniques du cheptel bovin est essentielles pour l'élaboration des stratégies de développement appropriées et l'intensification durable de la production laitière en Afrique de l'Ouest. C'est dans cette optique que la présente étude est menée. L'objectif de l'étude est de déterminer les paramètres démographiques et zootechniques du cheptel bovin de la zone péri-urbaine de Bobo-Dioulasso, à l'Ouest du Burkina Faso. La méthode d'enquête rétrospective des douze derniers mois (12mo) a été utilisée. Il a concerné au total 64 troupeaux laitiers installés à la périphérie de la ville dans quatre campements riverains. Les résultats obtenus indiquent que la taille moyenne des troupeaux est de 46 têtes dont $70 \%$ de femelles. Les races Zébus peulh soudanien et les métis Méré sont les races dominantes avec respectivement $80 \%$ et $15 \%$. Les autres races notamment le Zébu Goudali, le Zébu M'Bororo, Zébu Azawak, le Gir et leurs métis ne représentent que 5\% du cheptel enquêté. Le taux de mise bas pour les 12 mois qui ont précédés l'enquête varie entre $46,77 \%$ et $56,35 \%$ pour l'ensemble des troupeaux. Le taux de mortalité global est en moyenne de 6,85\% ; il est relativement plus élevé chez les veaux (24,82\%). Le taux d'exploitation global est de $13,20 \%$ dont $22,70 \%$ pour les mâles et de $9,71 \%$ pour les femelles. Les performances des troupeaux enquêtés sont caractéristiques des troupeaux laitiers extensifs en voix d'intensification.

(C) 2019 International Formulae Group. All rights reserved

Mots clés: Enquête 12mo, paramètres zootechniques, élevage péri-urbain, zone subhumide, Burkina Faso.

\section{Analyses of demographic and zootechnic parameters of peri-urban dairy herd of Bobo Dioulasso, Burkina Faso}

\begin{abstract}
Information on the demographic and zootechnic parameters of cattle herds is key for designing appropriate development and sustainable intensification of dairy sector in West Africa. For this purpose, we conducted this study which aim is to assess the demographic and zootechnic parameters of the peri-urban dairy cattle herds in Bobo Dioulasso in western Burkina Faso. The 12 months' retrospectives herd survey method (12mo) was used. It covered in total, sixty-four (64) dairy herds in the peri-urban area of the city from four livestock keepers' camps. Results indicated that average herd size is 46 heads of cattle with $70 \%$ of females
\end{abstract}


dairy cows. Zebu peulh Soudanien (also called Fulani Sudanese Zebu) and it crossed breeds with taurin breeds (called Méré) were the dominants breeds kept, representing about $80 \%$ et $15 \%$ of the total herd, respectively. Other breeds such as Zebu Goudali, Zebu M'Bororo, Zebu Azawak, Gir and their crossed breeds cows represent only $5 \%$ of the herds monitored. Birth rate of the 12 months preceding the survey varies from $46.77 \%$ to $56.35 \%$. Average global mortality rate was estimated about $6.85 \%$ but mortality rate for youth animals was higher $(24.82 \%)$. Global off take rate is estimated about $13.20 \%$ with higher rate for male $(22.70 \%)$ as compared to females $(9.71 \%)$. Our findings show that the peri-urban herds monitored are in extensive dairy production systems towards intensification.

(C) 2019 International Formulae Group. All rights reserved

Keywords: 12 mo Survey, demographic and zootechnic parameters, peri-urban livestock, sub humid zone, Burkina Faso.

\section{INTRODUCTION}

L'élevage constitue un secteur important dans l'économie denombreux pays de l'Afrique de l'Ouest, particulièrement les pays sahéliens comme le Burkina Faso, le Niger et le Mali. Dans ces pays, le soussecteur de l'élevage contribueprès de $44 \%$ au PIB agricole régional, et joue un rôle socioéconomique important dans la lutte contre la pauvreté (CEDEAO, 2009), ainsi que l'atteinte de la sécurité alimentaire et nutritionnelle des ménages. Au Burkina Faso, l'élevage contribue pour plus de $18 \%$ à la formation du PIB et représente près de $26 \%$ des exportations du pays (MRA, 2010). Le cheptel bovin du pays a été estimé en 2014 à plus de 9 millions de têtes avec un taux de croît annuel de $2 \%$ et un taux d'exploitation de 12\% (MRA, 2015). L'activité d'élevage est pratiquée par plus de $80 \%$ des ménages ( $92 \%$ étant du milieu rural) qui en tirent tout ou une partie de leurs revenus (MRA, 2010). L'élevage des ruminants domestiques (bovins, ovins, caprins) au Burkina Faso est dominé par le système extensif (Kagoné, 2001) de type pastoral transhumant et agropastoral. On rencontre aussi des exploitations intensives qui se développent autour des filières bétailviande et lait notamment dans les grands centres urbains et leurs périphéries. Le système extensif fournit près de $90 \%$ de la production nationale de viande et $95 \%$ de la production nationale de lait frais (MRA, 2010), malgré la faible productivité par tête des vaches laitières utilisées. Dans ces systèmes extensifs et semi intensifs, l'alimentation $\mathrm{du}$ bétail est basée sur l'exploitation des pâturages naturels et des résidus de récoltes, avec une faible supplémentation en saison sèche à travers l'utilisation des sous-produits agro industriels comme le tourteau de Coton, les sons de céréales et d'autres concentrés. La précarité alimentaire, notamment l'insuffisance des ressources alimentaires en saison sèche et leur faible qualité, ainsi que la recrudescence de certaines maladies animales sont les principales causes majeures de la faible performance des troupeaux bovins. Les crises climatiques passées (notamment les sècheresses des années 70 et 80 ) et la variabilité spatio temporelle de la pluviométrie ainsi que l'accroissement de la population ont favorisé la modification de la géographie de l'élevage à travers le déplacement et l'installation des éleveurs des régions sahéliennes vers la zone soudanienne plus humide (Sawadogo, 2011) avec leurs troupeaux, où les ressources pastorales sont plus ou moins abondantes. De plus, l'accroissement démographique et l'urbanisation croissanteont fait des grands centres urbains du pays comme BoboDioulasso des pôles d'attraction des populations (Coulibaly, 2008). Ces villes offrent des opportunités de marché aux produits d'élevage, ce qui accentue la sédentarisation des éleveurs autour de ces villes malgré la forte pression foncière. Ces différents changements notamment le 
processus de sédentarisation des éleveurs autour des centres urbains et dans les zones subhumides, la pression foncière et l'ouverture au marché contribuent à la modification des caractéristiques structurelles des troupeaux bovins des zones péri-urbaines (Coulibaly, 2008). Bien que des études antérieures aient investiguées les performances des élevages laitiers périurbains en Afrique (Coulibaly, 2008; Ba et al., 2009 ; Toko et al., 2016), il n'en demeure pas moins que la connaissance de la dynamique des effectifs ainsi que les paramètres démographiques du cheptel bovin en zone péri-urbaine est nécessaire pour l'élaboration de stratégies de développement et d'intensification de l'élevage laitiers périurbains pour répondre à la demande des villes en lait et produits dévirés du lait. C'est dans cette optique que la présente étude a été conduite. L'objectif global est de déterminer les paramètres démographiques du cheptel bovin de la zone péri-urbaine de la ville de Bobo-Dioulasso. Plus spécifiquement, il s'est agi de caractériser la structuredes troupeaux bovins laitiers et d'estimer certains paramètres démographiques et zootechniques dans des élevages péri-urbains de la ville de BoboDioulasso.

\section{MATERIEL ET METHODES}

\section{Site de l'étude : localisation et caractéristiques biophysiques et humains}

L'étude a été menée dans la zone périurbaine de la ville de Bobo-Dioulasso dans la région des Hauts-Bassins à l'Ouest du Burkina Faso (Figure 1). Cette région est caractérisée par un climat de type Sud Soudanien avec une longue saison sèche (octobre à avril) et une saison pluvieuse d'une durée de cinq (05) mois (mai à septembre). La saison sèche se compose d'une période froide (novembre à janvier) et d'une période chaude (février à avril). La température moyenne est de $27^{\circ} \mathrm{C}$ avec des minimas de $20^{\circ} \mathrm{C}$ en décembre et des maximas de $35^{\circ} \mathrm{C}$ en avril. Les pluies sont relativement abondantes mais inégalement réparties dans le temps et dans l'espace. La pluviométrie varie entre 900 et $1250 \mathrm{~mm}$ d'eau par an. La végétation est constituée de savanes boisées, arborées et arbustives. En ce qui concerne la population, au recensement de 2006, il a été dénombré dans la région des Hauts-Bassins 1.490.942 habitants dont 497.191 dans la ville de BoboDioulasso (Badolo, 2009). La population connaît une augmentation remarquable. Cette augmentation démographique est en partie d'origine migratoire des populations venues de la zone Nord du pays. Le secteur primaire (agriculture et élevage) reste le secteur d'activité le plus représenté $(69,3 \%$ de la population active) dans la région au vu des conditions climatiques favorables.

\section{Échantillonnage et méthode d'enquête}

Pour les enquêtes et le suivi des troupeaux, le campement Benkadi du secteur 21 de la ville, le campement peulh de Nasso, le village de Diaradougou, de Bama et de Koumbia ont été sélectionnés sur la base des critères suivants : proximité avec la ville de Bobo Dioulasso, présence d'éleveurs pasteurs et agropasteurs peulhs sédentarisés, troupeaux non transhumants pendant la phase d'enquête et éleveurs organisés en groupement et/ou associations.Les troupeaux bovins d'un échantillon de 64 éleveurs essentiellement pastoraux et agropastoraux de la zone périurbaine de la ville de Bobo-Dioulasso ont été enquêtés du 8 décembre 2016 au 23 janvier 2017. Le choix des troupeaux enquêtés a été fait de manière aléatoire parmi les éleveurs retenus après une phase pré-enquête au cours de laquelle des données qualitatives sur les systèmes de production ont été collectées.

La méthodologie d'enquête utilisée dans le présent travail a été décrite par Lesnoff et al. (2007). Il s'agit de la méthode d'enquête rétrospective "des douze derniers mois" (12mo), qui a permis de dégager les caractéristiques démographiques des cheptels (taille et structure des troupeaux, reproduction, mortalité, exploitation). Les performances de production annuelle telles 
que les taux de mise bas de mortalité et d'exploitation ont été calculées avec les formules établies par Lesnoff (2011).

Taux de mise bas $=$ nombre de mise bas $\mathrm{x}$ 100/nb. Femelles reproductrices

Taux de mortalité $=$ nombre total de morts $\mathrm{x}$ 100/ effectif du troupeau

Taux d'exploitation $=$ nombre d'animaux d'exploités x 100/ effectif du troupeau

\section{Analyses statistiques}

Les données collectées ont été vérifiées et saisies dans la base de données Access du model 12mo puis importées dans le tableur EXCEL version 2013 pour calculer les différents paramètres démographiques. Une analyse comparée par site a été faite grâce au logiciel XLSTAT version 2007. Le test non paramétrique de Kruskal-Wallis (variables la taille du troupeau, taux de mortalité et taux d'exploitation) et le test de Fisher (variables structure du troupeau et taux de mise bas) ont été utilisés pour ces différentes analyses comparées au seuil de $5 \%$.

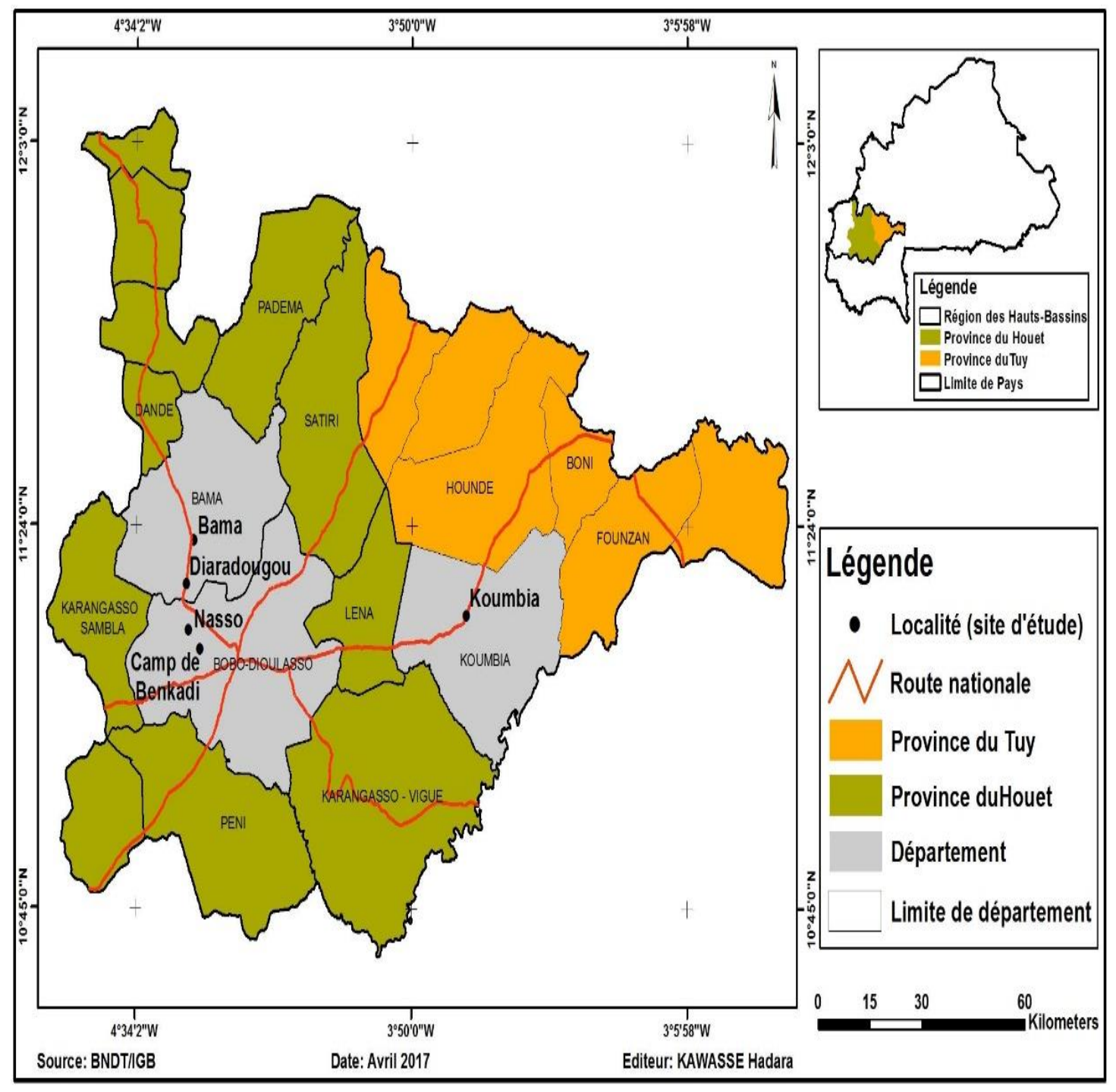

Figure 1: Carte de localisation des différents sites d'étude. 
RESULTATS

\section{Caractéristiques démographiques des troupeaux}

Taille moyenne et structure des troupeaux

Sur l'ensemble des 64 troupeaux enquêtés, les effectifs varient entre 17 et 103 têtes avec une taille moyenne de 46 têtes (Tableau 1). La structure par sexe des troupeaux bovins enquêtésindique une très forte proportion de femelles de l'ordre de $70 \%$ dont 53\% de vaches en âges de reproduction ( $\geq 4$ ans). La proportion des juvéniles (0 - 1 an) et d'adultes ( $\geq 4$ ans) est respectivement de l'ordre de $19 \%$ et $43 \%$ dans la zone d'étude. Alors que celle des sub-adultes ( 1 - 4 ans) varie entre $33 \%$ et $43 \%$. Excepté la proportion des sub-juvéniles dont une différence significative est observée entre sites, la proportion des juvéniles et adultes sont similaires à travers les sites.

\section{Principales races bovines gardées dans les élevages de la zone d'étude}

Les principales races bovines rencontrées dans les troupeaux suivis sont la race Zébu Peul Soudanien $(80,14 \%)$ et leur métis localement appelé Méré (15,58\%)
(Métis issus du croisement Zébu Peulh et taurins). Les zébus Goudali, Gir, M'Bororo et Azawak ainsi que leurs métis (métis issus de leur croisement avec les races locales) bien que rencontrées dans certains élevages suivis représentent que moins de 5\% des races gardées par les éleveurs (Figure 2).

\section{Estimation des paramètres de reproduction et d'exploitation des troupeaux}

Les performances de reproduction des troupeaux enquêtés sont en général faibles avec un taux moyen de mise bas de $51,69 \%$.Dans la zone d'étude, la mortalité est en moyenne de $6,85 \%$. Suivant les classes d'âge, ce taux de $24,82 \%$ chez les juvéniles (0-1an), de 5,3\% pour les sub-adultes (1-4ans) et de $4,89 \%$ chez les adultes ( $\geq 4 \mathrm{ans}$ ).Le taux d'exploitation global tous sexes et sites confondus est de 13,20\%. Mais celui des mâles est supérieur à celui des femelles et cela dans tous les sites (Tableau 2).

Tableau 1 : Taille moyenne et structure des troupeaux bovins de la zone périurbaine de la ville de Bobo-Dioulasso.

\begin{tabular}{|c|c|c|c|c|c|c|c|}
\hline \multirow[b]{2}{*}{ Sites d'étude } & \multirow{2}{*}{$\begin{array}{l}\text { Nbre de } \\
\text { troupeaux } \\
\text { enquêtés }\end{array}$} & \multirow[b]{2}{*}{$\begin{array}{l}\text { Taille } \\
\text { moyenne }\end{array}$} & \multicolumn{2}{|c|}{ Structure par sexe (\%) } & \multicolumn{3}{|c|}{ Structure par classe d'âge (\%) } \\
\hline & & & Mâles & Femelles & $\begin{array}{l}\text { Juvéniles } \\
\text { (0-1an) }\end{array}$ & $\begin{array}{l}\text { Sub-adultes } \\
\text { (1-4 ans) }\end{array}$ & $\begin{array}{l}\text { Adultes } \\
\text { ( } \geq \text { 4ans) }\end{array}$ \\
\hline Bama & 13 & $58 \mathrm{a}$ & $28 \mathrm{a}$ & $72 a$ & $22 \mathrm{a}$ & $35 \mathrm{ab}$ & $43 \mathrm{a}$ \\
\hline C. Benkadi & 17 & $40 \mathrm{a}$ & $29 a$ & $71 \mathrm{a}$ & $17 \mathrm{a}$ & $43 \mathrm{a}$ & $40 \mathrm{a}$ \\
\hline Diaradougou & 9 & $50 \mathrm{a}$ & $31 \mathrm{a}$ & $69 a$ & $19 \mathrm{a}$ & $33 b$ & $48 \mathrm{a}$ \\
\hline Koumbia & 14 & $41 \mathrm{a}$ & $30 \mathrm{a}$ & $70 \mathrm{a}$ & $18 \mathrm{a}$ & $38 \mathrm{ab}$ & $44 \mathrm{a}$ \\
\hline Nasso & 11 & $41 \mathrm{a}$ & $33 \mathrm{a}$ & $67 \mathrm{a}$ & $21 \mathrm{a}$ & $37 \mathrm{ab}$ & $42 \mathrm{a}$ \\
\hline Total & 64 & 46 & 30 & 70 & 19 & 38 & 43 \\
\hline
\end{tabular}

$\mathrm{a}, \mathrm{b}$ : les valeurs moyennes sur la même colonne avec les mêmes lettres ne sont pas significativement différentes à 5\% $(\mathrm{P} \geq 0,05)$. 


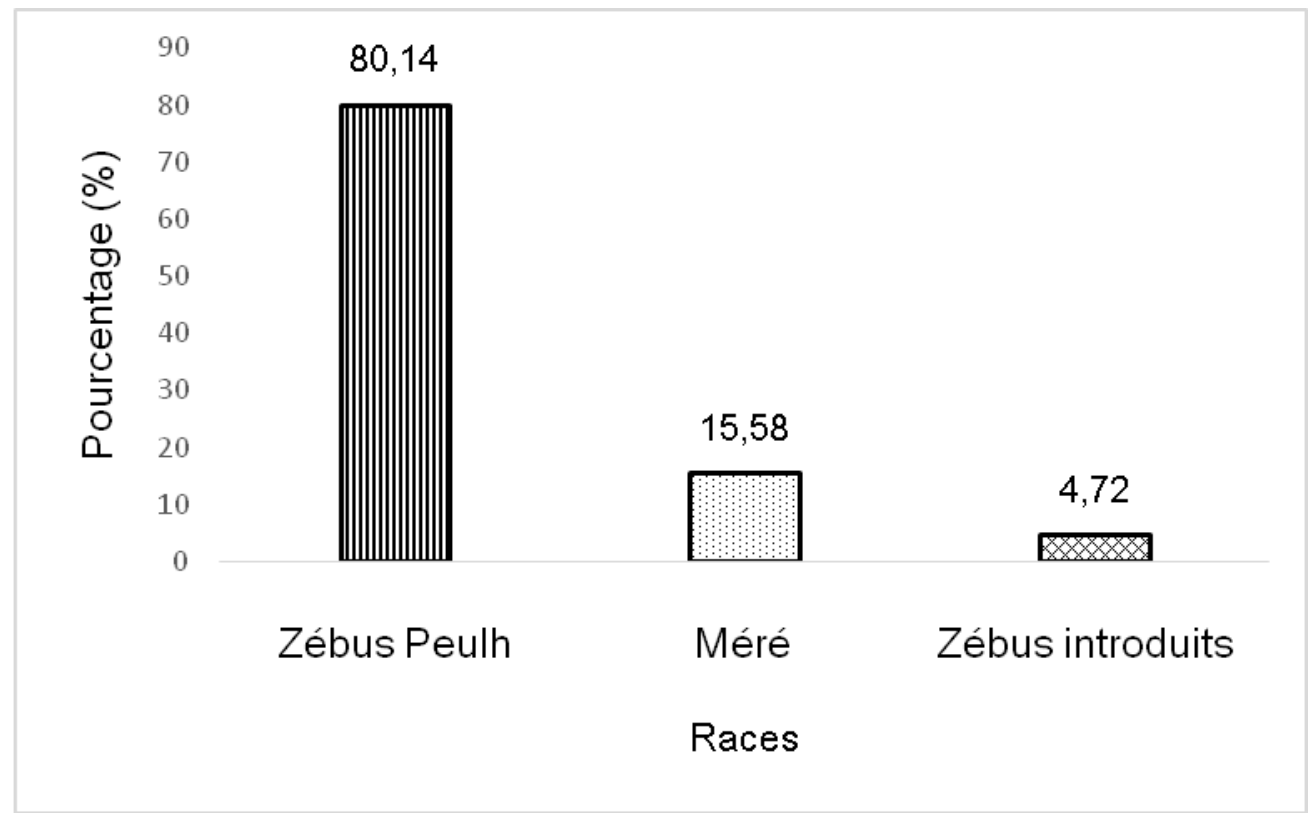

Figure 2 : Races bovines constituant les troupeaux suivis.

Tableau 2 : Paramètres de reproduction et de gestion des troupeaux bovins.

\begin{tabular}{|c|c|c|c|c|c|c|c|c|}
\hline \multirow{3}{*}{ Site d'étude } & \multirow{3}{*}{$\begin{array}{l}\text { Taux de } \\
\text { mise bas }\end{array}$} & \multicolumn{4}{|c|}{ Taux de mortalité } & \multicolumn{3}{|c|}{ Taux d'exploitation } \\
\hline & & \multicolumn{3}{|c|}{ Classe d'âge } & \multirow[t]{2}{*}{ Global } & \multirow{2}{*}{ Mâles } & \multirow{2}{*}{ Femelles } & \multirow{2}{*}{ Global } \\
\hline & & Juvéniles & Sub-adultes & Adultes & & & & \\
\hline Bama & $56,35 \mathrm{a}$ & $9,30 \mathrm{a}$ & $8,24 \mathrm{a}$ & $2,24 \mathrm{a}$ & $4,91 \mathrm{a}$ & $19,22 \mathrm{a}$ & $12,07 \mathrm{a}$ & $12,76 \mathrm{a}$ \\
\hline C. Benkadi & $49,39 \mathrm{a}$ & $30,66 a$ & $4,37 \mathrm{a}$ & $7,33 \mathrm{a}$ & $9,43 a$ & $30,68 \mathrm{a}$ & $10,48 \mathrm{a}$ & $15,96 a$ \\
\hline Diaradougou & $52,54 \mathrm{a}$ & $57,66 \mathrm{a}$ & $5,00 \mathrm{a}$ & $4,36 \mathrm{a}$ & $8,47 \mathrm{a}$ & $12,39 \mathrm{a}$ & $8,82 \mathrm{a}$ & $9,45 \mathrm{a}$ \\
\hline Koumbia & $46,77 \mathrm{a}$ & $14,06 \mathrm{a}$ & $5,49 \mathrm{a}$ & $10,27 \mathrm{a}$ & $6,90 \mathrm{a}$ & $30,04 a$ & $9,39 a$ & $15,36 \mathrm{a}$ \\
\hline Nasso & $55,32 \mathrm{a}$ & $12,29 \mathrm{a}$ & $3,51 \mathrm{a}$ & $0,85 \mathrm{a}$ & $4,49 \mathrm{a}$ & $16,98 \mathrm{a}$ & $7,32 \mathrm{a}$ & $10,95 \mathrm{a}$ \\
\hline Total & 51,69 & 24,82 & 5,53 & 4,89 & 6,85 & 22,70 & 9,71 & 13,20 \\
\hline
\end{tabular}

NB: les valeurs moyennes sur la même colonne avec les mêmes lettres ne sont pas significativement différentes à 5\% $(\mathrm{P} \geq 0,05)$. 


\section{DISCUSSION}

\section{Taille et structure des troupeaux suivis}

Les résultats de l'étude ont révélé une relative grande taille des troupeaux suivis, qui est caractéristique des troupeaux pasteurs et agropastoraux de l'Afrique de l'Ouest malgré la sédentarisation des éleveurs à la périphérie de la ville. En effet, dans ces types d'élevage, la taille du troupeau représente la capacité de production de veaux, de lait, de viande ou encore de force de travail du troupeau ayant une valeur monétaire pour l'éleveur. Elle représente ainsi donc le capital économique de l'éleveur et joue un rôle socio-culturel dans les communautés pastorales et agropastorales. Les bovins constituent une épargne mobilisable en cas de nécessité majeure. La constitution de troupeaux de grands effectifs serait une stratégie de résilience pour ces éleveurs face aux chocs climatique. Les effectifs élevés leur permettraient d'assurer un certain niveau de vie et de préserver le troupeau des fortes mortalités dues à la sècheresse. Des résultats similaires ont été reportés par Toko et al. (2016) qui ont obtenus une taille moyenne de 66 têtes dans les élevages peulhs au Nord du Benin. Otte et Chilonda (2002) estime qu'en Afrique Subsaharienne, la taille moyenne des troupeaux, varie entre 15 à 157 têtes. Mais il faut noter que la taille des troupeaux bovins dans les systèmes pastoraux et agropastoraux est soumise à des fluctuations et à des variations interannuelles dues au système d'élevage, aux conditions climatiques et environnementales. Une taille moyenne de 70 têtes a été obtenue par Adanléhoussi et al. (2005) dans les élevages traditionnels périurbains de BoboDioulasso, qui est supérieur à celle obtenue dans cette étude (46 têtes de bovins).

Pour ce qui est de la structure des troupeaux suivis, elle est caractéristique des troupeaux naisseurs orientés dans la production laitière vue la proximité avec la ville et la demande en produits laitiers des citadins. En effet, une structure démographique, où la proportion des reproductrices tourne autour de $40 \%$ indique la spécialisation des troupeaux dans la production laitière (Kassa et al., 2016). La grande proportion de vaches reproductrices et des génisses déterminerait la rentabilité de l'élevage. Plus il y a de vaches reproductrices plus il y a du lait et des veaux dont la commercialisation génère des revenus pour le ménages. De plus, les génisses assurent le renouvellement du troupeau. Nos résultats sont dans le même ordre de grandeur que ceux obtenus Adanléhoussi et al. (2005) qui ont estimé à $63 \%$ la proportion de femelles dans les élevages traditionnels de la zone périurbaine de Bobo-Dioulasso. La grande proportion de jeunes (juvéniles et sub-adultes) dans les troupeaux serait également une caractéristique de troupeaux en reconstitution.

\section{Races bovines gardées dans les élevages péri-urbains de Bobo Dioulasso}

La prédominance des Zébus Peulhs soudanien (trypano sensibles) dans les élevages de la zone d'étude pourrait s'expliquer d'une part par les efforts de lutte efficace contre la glossine et d'autre part par la disponibilité des trypanocides sur le marché facilitant les traitements des animaux. Ce qui permettrait aujourd'hui à cette race non trypano-tolérante d'être élevée dans la zone subhumide. La proportion non négligeable de Métis (Méré) rencontrée dans les différents sites indique un croisement entre zébu peulh et la race taurine par des éleveurs qui sont plus ou moins orientés vers l'obtention d'animaux plus performants pour la traction animale, tout en conservant un minimum de tolérance vis-àvis de la trypanosomiase (FAO, 2005). La présence des autres races d'autres régions d'Afrique et d'Europe (essentiellement constitué de zébus) bien que minoritaires indiquerait une volonté des éleveurs orientés vers la production laitière d'améliorer les performances laitières de leurs troupeaux à travers l'insémination artérielle et l'importation de races laitières. La proximité de la ville de Bobo-Dioulasso peut être un facteur influençant cette stratégie, afin de satisfaire les demandes en produits animaux (lait et viande). Cette introduction aurait aussi été favorisée par les différents projets et 
programmes d'amélioration génétique à travers l'importation des races exotiques, le croisement entre races locales et races exotiques (insémination artificielle) aussi bien au niveau de la recherche notamment le CIRDES et ses partenaires ainsi que le ministère des ressources animales et halieutiques du Burkina Faso. Gnanda et al. (2016) remarquent que dans les élevages périurbains bien que la saillie naturelle soit le principal mode de reproduction utilisé par les exploitants, l'insémination artificielle y est aussi associée.

\section{Performances reproductive des animaux et exploitation des troupeaux}

Les faibles performances de reproduction des troupeaux de la zone périurbaine de Bobo-Dioulasso sont généralement la conséquence à une alimentation déficitaire et des problèmes de santé. La forte pression agricole et démographique, de même que l'urbanisation grandissante rendent très contraignant l'accès aux ressources pastorales dans la zone d'étude. Les éleveurs dans les différents sites ont pratiquement les mêmes pratiques d'élevage, ce qui explique la similarité des taux de mise bas. Les performances de reproduction estimées par l'enquête $12 \mathrm{mo}$ dans cette étude sont similaires aux valeurs rapportées dans la littérature pour les troupeaux bovins en milieu sahélien et soudanien. Corniaux et al. (2012) ont rapporté qu'en Afrique de l'Ouest, les taux de mise bas des bovins sont généralement compris entre 45 et 55\%. Des résultats similaires ont été trouvés en Côte d'Ivoire (52\%), dans le Ferlo au Sénégal (45\%) et dans la zone de Sikasso au Mali (53\%) respectivement par Knopf et al. (2004), Jorat (2011) et Ba et al. (2009). Alkoiret et al. (2010) ont obtenu des taux de mise bas supérieurs $(70 \%)$ à nos résultats. Le suivi sanitaire des animaux, leur alimentation, la conduite du troupeau, ainsi que la méthode de collecte de données (suivi de troupeaux) seraient les facteurs de variation possible pouvant expliquer cette différence.

De façon générale, les élevages de la zone périurbaine de Bobo-Dioulasso sont caractérisés par un taux de mortalité moyen. Mais cette mortalité est élevée surtout chez les veaux (juvéniles). Les mortalités dans les troupeaux sont dues le plus souvent au déficit nutritionnel en saison sèche mais aussi au parasitisme pendant l'hivernage. En effet les causes de mortalité les plus citées par les éleveurs sont les déficits fourragers de la saison sèche, la trypanosomose (Bouubal, Soumaya en Foulfouldé et en Dioula) et la fièvre aphteuse ou Safa en Dioula. Youssao et al. (2000) nous ont rapporté que la sousalimentation, l'absence ou l'insuffisance des prophylaxies sanitaires appropriés et médicales sont les principales causes de mortalité en élevage traditionnel notamment pasteurs et agropasteurs (Otte et Chilonda, 2002 ; Corniaux et al., 2012 ; Youssao et al., 2013). Aussi le prélèvement d'une partie du lait pour la consommation humaine et la vente ont également des conséquences négatives sur la survie des veaux. Avec les opportunités de marché offertes par la ville de BoboDioulasso, les éleveurs périurbains seraient de plus en plus ouverts et attirés par la vente des animaux. Ce qui explique le taux d'exploitation élevé obtenu dans cette étude comparativement au taux national de $12 \%$ estimé par le MRA (2015) du Burkina Faso. Quel que soit le site considéré, le taux d'exploitation des mâles est supérieur à celui des femelles. Les élevages suivis étant de type naisseur, les mâles (taurillons) sont vendus très tôt alors que les femelles sont gardées pour la reproduction. Les éleveurs conservent les génisses, qui constituent leur capital laitier. Ces résultats corroborent avec ceux obtenus par Youssao et al. (2000) et par Alkoiret et al. (2010). Dans les troupeaux suivis, un mâle aurait $22,70 \%$ de chance d'être exploité tandis que pour une femelle cette chance serait de 9,71\%. D'après l'annuaire statistique du MRA (2015), les taux d'exploitation des mâles et des femelles au Burkina Faso sont estimés respectivement à $6,2 \%$ et $5,3 \%$. Ces taux sont largement inférieurs à nos résultats. Cela serait probablement dû à la méthode d'estimation utilisée par les services statistiques du ministère de l'élevage. De plus, leurs taux d'exploitation sont issus de données 
nationales. D'après Jorat (2011), l'exploitation reste sensiblement la même quelles que soient les variations des taux naturels. Mais il note que plus le taux de mortalité est faible plus le taux d'exploitation est élevé et vice-versa. Cependant, nos résultats indiquent le contraire. En effet, les sites Benkadi et Koumbia qui ont enregistré de forts taux de mortalité les plus élevé disposent également de fort taux d'exploitation (respectivement 15,96\% et $15,36 \%$ ). Une forte production (fort taux de mise bas) des troupeaux les années antérieures ou un déficit alimentaire au cours de la saison sèche (exploitation afin d'éviter les pertes) peut entraîner un taux d'exploitation élevé dans ces sites pour l'année 2016. La vente de taurillons de trait, les contrats et prêts de labours constituent l'essentiel des exploitations des animaux. Dans les différents sites, les femelles vendues ou exploitées sont pour la plupart de vieilles vaches réformées ou en fin de carrière. Dans les élevages pasteurs et agropasteurs, les abattages et les dons sont ponctuels et sont observés lors des cérémonies de mariage, de baptêmes, funérailles, fêtes religieuses, etc. (Youssao et al., 2000).

\section{Conclusion}

La présente étude contribue à une meilleure connaissance des caractéristiques structurelles et démographiques des élevages bovins laitiers péri-urbains de la ville de Bobo Dioulasso. Les résultats indiquent que les troupeaux de ces élevages sont caractérisés par une forte proportionde femelles et une taille moyenne de 46 têtes par troupeaux. La plupart des troupeaux sont naisseurs avec une orientation pour la production laitière vue la proximité avec la ville et les opportunités de marchés pour les produits animaux et d'originales comme le lait et la viande. Mais on enregistre un faible taux de mise bas et un taux de mortalité élevé surtout chez les juvéniles qui affectent les performances reproductives et de production de troupeaux. Les taux d'exploitation relativement élevé par rapport à la moyenne nationale s'expliquent par la proximité de la ville qui offre plus d'opportunités de marchés pour le bétail mais aussi le prélèvement des taurions pour vendre pour couvrir certains besoins du ménage mais aussi pour l'achat des SPAI et autres intrants vétérinaires. En guise de perspective, une caractérisation génétique plus approfondie des différentes races bovines par la biologie moléculaire permettront de mieux caractériser le potentiel génétique des races gardées dans les différents troupeaux. D'autres investigations sur les préférences des éleveurs des différentes races bovines utilisées ainsi que leurs connaissances en matière de gestion des ressources génétiques locales pourraient être utiles aux programmes d'amélioration et de valorisation des ressources génétiques animales.

\section{CONFLIT D'INTERETS}

Les auteurs déclarent n'avoir aucun conflit d'intérêts.

\section{CONTRIBUTION DES AUTEURS}

$\mathrm{NZ}$ a conçu le protocole de l'étude, superviser la collecte des données, analyser les données collectées et contribuer à la rédaction du manuscrit. IS est l'étudiant qui a contribué à la collecte des données et la rédaction du manuscrit de l'article. MS a contribué à la révision du protocole, à la rédaction et correction du manuscrit.

\section{REMERCIEMENTS}

Cette étude a été réalisée dans le cadre $\mathrm{du}$ project de recherches postdoctorale dénommé « Livestock production systems in the defined pastoral zones around protected forest areas in Burkina Faso : toward socioeconomic transformations of pastoralist communities' livelihood and livestock husbandry practices » (Az 88 0888) financé par la Fondation Volkswagen (en Allemagne). C'est l'un des projets de l'initiative Knowledge for tomorrow de la Fondation Volkswagen. Les auteurs voudraient remercier les éleveurs de la zone péri-urbaine de Bobo Dioulasso qui ont acceptés participer à cette étude. Les remerciements également à Traoré Tidiane, Sarambé Cécile et Kawassé Hadara, du CIRDES (Centre de Recherche Développement de l'élevage en Zone 
subhumide) qui ont aussi participé à la collecte des données.

\section{REFERENCES}

Adanléhoussi A, Hamadou S, Hamani M, Kamuanga M, Salissou I, Sangaré M, Tamboura HH. 2005. Caractérisation des systèmes de production laitière en zone CIRDES. In: Rapport final PROCORDEL, CIRDES-ILRI, BoboDioulasso, Burkina Faso, 86-94.

Alkoiret TI, Awohouedji DYG, Yacoubou AM. 2010. Paramètres démographiques des cheptels de bovins Borgou et N'Dama à la Ferme d'Elevage de l'Okpara au nord-est du Bénin, International Journal of Biological and Chemical Sciences, 4(5): 1657-1666.

Ba A, Lesnof M, Coulibaly D, ChapuisPoccard R, Moulin C. 2011. Un outil simple de projection démographique pour estimer la productivité d'un cheptel : application à un cheptel bovin de la zone cotonnière au Mali-Sud, Actes du séminaire, Bobo-Dioulasso, Burkina Faso, 8 p.

Ba A, Lesnoff M, Poccard-chapuis R., Corniaux, Moulin CH. 2009. Évaluation du potentiel exploitable du cheptel de bovins dans la zone cotonnière du Mali. $16^{\text {ème }}$ journées Rencontres Recherches Ruminants, $16: 385$.

Badolo H. 2009. Monographie de la région des Hauts-Bassins, Recensement général de la population et de L'habitation (RGPH) de 2006. Ministère de l'Économie et des Finances, Ouagadougou, Burkina Faso, 154 p.

CEDEAO (Communauté Economique des Etats d'Afrique de l'Ouest). 2009. Sousprogramme gestion améliorée des autres ressources naturelles partagées. Formulation et mise en cuvre d'un Programme Régional d'Investissement Agricole (PRIA), Composante : " Aménagements pastoraux et organisation de la transhumance transfrontalière volume 2 (version finale). CEDEAO, 58 p.
Corniaux C, Lesnoff M, Ickowicz A, Hiernaux P, Diawara MO, Sounon A, Aguilhon M, Dawalak A, Manoli C, Assani B, Jorat T, Chardonnet F. 2012. Dynamique des cheptels de ruminants dans les communes de Tessékré (Sénégal), Hombori (Mali), Dantiandou (Niger) et Djougou (Bénin). ANRECLIS, $41 \mathrm{p}$.

Coulibaly D. 2008. Changements sociotechniques dans les systèmes de production laitière et commercialisation du lait en zone péri-urbaine de Sikasso, Mali. Thèse de Doctorat de l'Institut des Sciences et Industries du vivant et de l'Environnement (Agro Paris Tech), Ecole doctorale Institut National Agronomique Paris-Grignon, France, $392 \mathrm{p}$.

Gnanda BI, Bado D, Reusse SPare L. 2016. Caractéristiques et typologie des exploitations laitières bovines de la zone périurbaine de Ouagadougou, au Burkina Faso. $23^{\text {ème }}$ journées Rencontres Recherches Ruminants, 165-168.

Jorat T. 2011. Simulations de dynamiques de cheptels bovins après une sècheresse au sahel en fonction des types d'exploitation pastorales : cas du Ferlo au Sénégal, Rapport de deuxième année Master BGAE-Sciences pour l'environnement (spécialité Ecologie fonctionnelle et développement durable parcours EPSED Elevage des pays du sud : Environnement, Développement), CIRAD, $53 \mathrm{p}$.

Kagoné H. 2001. Profil fourrager du Burkina Faso. FAO, 23 p.

Kassa KS, Ahounou S, Dayo GK, Salifou C, Issifou MT, Dotche I, Gandonou PS, Yapi-Gnaore V, Koutinhouin B, Mensah GA, Youssao IAK. 2016. Performances de production laitière des races bovines de l'Afrique de l'Ouest. Int. J. Biol. Chem. Sci., 10(5): 2316-2330. DOI: http://dx.doi.org/10.4314/ijbcs.v10i5.29

Knopf L, Komoin-Oka C, Betschart B, Gottstein B, Zinsstag J. 2004. Production and Health Parameters of N'Dama Village Cattle in Relation to Parasitism 
in the Guinea Savannah of Côte d'Ivoire. Revue d'Elevage et de Médecine Vétérinaire des Pays Tropicaux, 57(1-2): 95-100.

Lesnoff M. 2011.Démographie et zootechnie tropicales : un lien par les modèles matriciels appliqués aux cheptels de ruminants dans les élevages extensifs. Mémoire de synthèse en vue d'une candidature à une habilitation à diriger des recherches, Université de Montpellier II, $221 \mathrm{p}$.

Lesnoff M, Saley M, Adamou K, N'Djaffa, H, Ayantunde A, Gerard B. 2007. 12mo : Une méthodologie rétrospective pour l'estimation des paramètres démographiques des cheptels de ruminants domestiques tropicaux. CIRAD-ILRI, $58 \mathrm{p}$.

MRA (Ministère des Ressources animales) 2005. Les statistiques du secteur de l'élevage au Burkina Faso. MRA, 61 p.

MRA (Ministère des Ressources animales) 2009. Document de Politique de développement de l'élevage au Burkina Faso 2010-2020. MRA, 40 p.

MRA (Ministère des Ressources animales) 2010.Politique nationale de développement durable de l'élevage au Burkina Faso 2010-2025. MRA, 54 p.

MRA (Ministère des Ressources Animales). 2015. Annuaires des statistiques de l'élevage 2014. MRA, $177 \mathrm{p}$.

Otte MJ, Chilonda P. 2002. Cattle and small ruminant production systems in subSaharan Africa: a systematic review. Livestock Information Sector Analysis and Policy Branch, FAO Agriculture Department, Rome, Italy, 98 p.
Sawadogo I. 2011. Ressources fourragères et représentations des éleveurs, évolution des pratiques pastorales en contexte d'aire protégée: Cas du terroir de Kotchari à la périphérie de la réserve de biosphère du $\mathrm{W}$ au Burkina Faso. Thèse de Doctorat (Spécialité : Physiologie et Biologie des Organismes- Populations Interactions), Ecole Doctorale Sciences de la Nature et de l'Homme, Museum National d'Histoire Naturelle, ParisFrance, $335 \mathrm{p}$.

Toko RC, Adégbidi A, Lebailly P. 2016. Démographie et performances zootechniques des élevages bovins traditionnels au Nord Bénin. Revue d'Elevage et de Médecine Vétérinaire des Pays Tropicaux, 69(1): 33-39.

Youssao AKI, Ahissou A, Touré Z, Leroy PL. 2000. Productivité de la race Borgou à la Ferme d'élevage de l'Okpara au Bénin. Revue d'Elevage et de Médecine Vétérinaire des Pays Tropicaux, 53(1): 67-74.

Youssao AKI, Dahouda M, Attakpa EY, Koutinhouin GB, Ahounou GS, Toléba SS, Balogoun BS. 2013. Diversité des systèmes d'élevages de bovins de race bovine Borgou dans la zone soudanienne du Bénin. Int. J. Biol. Chem. Sci., 7(1): 125-146. DOI: DOI: http://dx.doi.org/10.4314/ijbcs.v7i1i.11

FAO 2005. Trypanotolerant livestock in the context of trypanosomiasis intervention strategies. Paat technical and scientific series 7, Rome, Italy. 